\title{
Enabling Fast Ramp-Up of Assembly Lines through Context-Mapping of Implicit Operator Knowledge and Machine-Derived Data
}

\author{
Konstantin Konrad ${ }^{1}$, Michael Hoffmeister ${ }^{1}$, Matthias Zapp ${ }^{1}$, \\ Alexander Verl ${ }^{1}$, and Johannes Busse ${ }^{2}$ \\ ${ }^{1}$ Fraunhofer Institute for Manufacturing Engineering and Automation, Stuttgart, Germany \\ (Konstantin.Konrad, Michael.Hoffmeister, Matthias.Zapp, \\ Alexander.Verl) @ipa. fraunhofer. de \\ ${ }^{2}$ Johannes Busse Knowledge Engineering, Heidelberg, Germany \\ jbusseajbusse.de
}

\begin{abstract}
Ramp-up of precision assembly lines is a cost-intensive and experience-driven task. Most of the time the knowledge how to effectively and efficiently setup an assembly line is intrinsic and is therefore neither shared nor reused by production experts. Almost no machine data is recorded until the correct functionality of the line is achieved and human problem solving tasks are not or poorly documented. In this paper a novel approach for structuring operator knowledge and combining it with machine-derived data by the use of semantic technologies is proposed. This enables human operators to express their experience in an easy to understand, machine readable way and makes it therefore accessible to other workers.
\end{abstract}

Keywords: Semantics, Ontology, Assembly Line, Knowledge Management, Machine-Derived Data, Operators.

\section{Introduction}

In the literature different variants for ramp-up are considered, either the start of new products, the introduction of a new or the reorganization of an existing production system as well as all feasible combinations. One possible approach is defining the ramp-up time of products as the "time required from finishing prototyping to full volume production" [1]. However in this paper we are focusing on the ramp-up of new production processes and the resulting problem areas. Therefore ramp-up is defined as the phase between production development and the full capacity of production with a sufficient level of quality. Depending on the industrial application area ramp-up can take from a couple hours up to several years.

Approximately $82 \%$ of the companies do not reach their targeted goals regarding time, cost and quality [2]. This is very critical as volatile market requirements need a rapidly shortened time-tomarket [3]. Main reasons for missing the target goals are continuous changes at product and/or process level. Despite that a lot of companies do not make use of software solutions during their ramp-up procedure besides standard 
MES or ERP functionalities [2]. Successful companies have an overview about present key performance indicators (i.e. Overall Equipment Effectiveness) during the ramp-up or have proper simulation tools installed [4]. As matters of fact, small and medium enterprises (SMEs) do use these tools to an even lower extend due to cost factors and limited availability of human resources. For these SMEs a lack of coordination and communication is a major obstacle for a successful and fast ramp-up of assembly lines [2]. However these characteristics are especially important when SMEs concern about the high requirements for precision products.

Key for a successful ramp-up are therefore highly skilled and motivated human workers and the knowledge transfer from experience gained in former projects. Due to time pressure and upcoming deadlines the gathered experience is hardly ever documented in a proper way for reuse. At present, known knowledge management systems are hardly implemented and used during the ramp-up procedure [5].

Also Berger at al. [6] sees the consequent usage of experience gained about comparable assembly machines or lines as key for success. Many important observations and conclusions of production staff are simply lost and not reimplemented as improvements in consecutive projects. Fleischer et al. [7] sees the strong dependencies on intrinsic operator experience for troubleshooting as one of the main deficits in production ramp-up.

\subsection{Success Factors for Ramp-Up}

All this leads to the conclusion that for a successful ramp-up of assembly lines it is essential to gather the experience from production workers in an effective and efficient way and make it available also to others for re-usage and further refinement. The presented approach will target some major obstacles, which are relevant for many companies in order to perform a successful knowledge management during the rampup procedure:

- $\quad$ Easy gathering of operator knowledge. An optimal solution for the ramp-up phase would be to gather knowledge in a causal "if-then" relationship which would enable the further usage and automatic processing of the operator experience [6]. However, the presented approach will go beyond that and allow multiple, easy to use, understandable, machine-readable crossrelations between different entities. This facilitates the adequate capturing, transfer and usage of human intrinsic knowledge e.g. about tolerances, assembly issues and material dependencies.

- Flexible context-mapping of static and dynamic data: At present most knowledge capturing systems only provide static capabilities to model the knowledge (i.e. Wiki-systems are widely used in companies). Besides that machine data is mostly stored in predefined databases or data-warehouses. Therefore it is not capable of adequately modeling all possible relations and the system administration is limited to IT experts which can hardly react in a timely manner to the arising problems during ramp-up. We will present a novel approach to combine large quantities of sensor data, external software systems like Statistical Process Control (SPC) software and operator knowledge in a human-centered and flexible way. This is of special 
importance for precision assembly as due to the high product requirements plenty of sensor data has to be integrated.

- Extensibility \& Reusability: Due to the nature of the ramp-up process not all data providers are present from the beginning as e.g. sensors are connected and adjusted in a later stage. Also software systems like SPC or Condition Monitoring are typically installed at a later stage. Also consecutive assembly lines might be equipped with additional or other hardware entities. The already modeled experience should be used in these projects and be further refined. This is of high importance as saving potential through Knowledge Management Systems is particularly high when ramping a similar equipment or line and experiencing alike problems. Due to these reasons the suggested solution should be able to integrate different data sources and apply modifications in an iterative manner. Also the modeled knowledge should be (partially) exportable for re-usage in other projects or for other operators.

\section{Approach and Methology}

\subsection{Semantic Technologies in Manufacturing}

In order to cope with the multitude of different data sources in modern production and the constantly changing environment of today's production lines, the application of "Semantic technologies" is intensively discussed in literature. These technologies try to provide an always-upto-date and highly structured access to a certain field of knowledge. According to Breslin et al. [8], such systems can be well-suited for industrial applications: blogs to effectively share information within organizations, collaborative document editing, support for project management and execution of software development, micro-blogging for fast information transport, semantic wiki systems to pursue knowledge management and semantic web and Web 2.0 approaches to effectively retrieve information from the internet. In addition to this, semantic web services are already addressing the complexity of data integration within industry [8]. The World Wide Web Consortium (W3C) provides an interesting collection of industrial use-cases within the industry [9].

The following sections will provide an overview on the underlying concepts of these technologies, the existing standards and the deployment of these capabilities to industrial environments.

\subsection{Ontologies}

Semantic technologies are building upon "ontologies". According to Gruber [10], a consensus definition is that an "ontology is a formal, explicit specification of a shared conceptualization". The conceptualization is applied towards a limited "domain", which for instance can be the ramp-up procedures of production lines. The conceptualization tries to break down the different terms and entities of this domain into well-defined and distinctive concepts. The concepts are expressed in a formal way in order to allow technical installations and software to use them. Further on, the concepts shall be explicit in order to avoid inconsistencies and differences in 
meaning; this can be achieved by using non-ambiguous classifications, relations or metrics. Lastly, the conceptualization shall be shared and agreed among different user groups in order to provide a means of communication and a frame of reference.

Applied to the field of ramping up micro-assembly lines, the use of ontologies opens possibilities to reach a joint understanding about important structural elements of a production line, by which procedures they are affected and how they correlate with different processes and measurement data. This will deepen the understanding about structure and data for all user roles concerned with the ramp-up, as e.g. the engineers designing the line, the software engineers creating the PLC programs as well as the engineers performing the ramp-up itself. All different user roles can use the same source of definition for performing their individual work tasks; boundaries are clearer defined. Looking at the ramp-up process as a whole, the use of ontologies can make the overall process more explicit and make it decomposable into several well-defined actions, which can be documented and compared with similar ramp-up situations. This breaks up the implicit understanding of a ramp-up process and exposes it to systematic knowledge management, which can be used to gather human knowledge and procedures, to interlink it with further information and to make it reusable for repeating situations.

Therefore, ideally, the use of ontologies can better organize the effective work of human engineers and, at the same time, they can be used to store and provide important information, process and measurement data.

\subsection{Semantic Middleware}

The creation and maintenance of ontologies can be performed in regular text editor by using an ontology language like Web Ontology Language (OWL). Alternatively, a number of dedicated ontology editors with graphical user interfaces such as Protégé [11] and OntoStudio [12] are available. Furthermore, as applied for this work, ontologies can also be defined and maintained via semantic annotated mindmaps, which are automatically transferred into an ontology language $[13,14]$.

In order to utilize the captured explicit knowledge in ontologies inside the production environment, these ontologies need to be incorporated in software systems. This can be realized in two different ways. One the one hand these functionalities can be directly incorporated into each individual software, e.g. by including libraries such as Jena [15], Jess [16] and Ontobroker [17]. One the other hand software applications can be connected against a central IT service, which provides the reasoning functionalities based on ontologies; such IT services are typically denoted as middleware. The OntoBroker middleware [18] which available as commercially supported product can be used as an embedded library or operated as a server application; therefore in the later configuration it has been used for this project.

\subsection{Standards for Semantic Technologies}

As production systems typically have a long life-time, the human knowledge and the acquired machine data from the ramp-up phase need to be persisted for an extended period of time. This is especially true, if ramp-up knowledge shall be re-used for 
subsequent versions of the production system. The reuse of knowledge also calls the need of merging knowledge and data from different sources, as new production systems might incorporate features from more than one prior system. This leads to high requirements for the maintainability, extensibility and scalability of semantic technologies and the need of using a set of well-founded standards, which guarantee accessibility of gained knowledge for a long time.

In the course of the foundation of the Semantic web [19], this need was early recognized by the $\mathrm{W} 3 \mathrm{C}$ consortium and a set of standards building on-top of each other, were specified. Semantic technologies, such as semantic middleware and wiki systems, are adhering to these standards. By complying with these, investments in central IT systems, software and personal training can be protected.

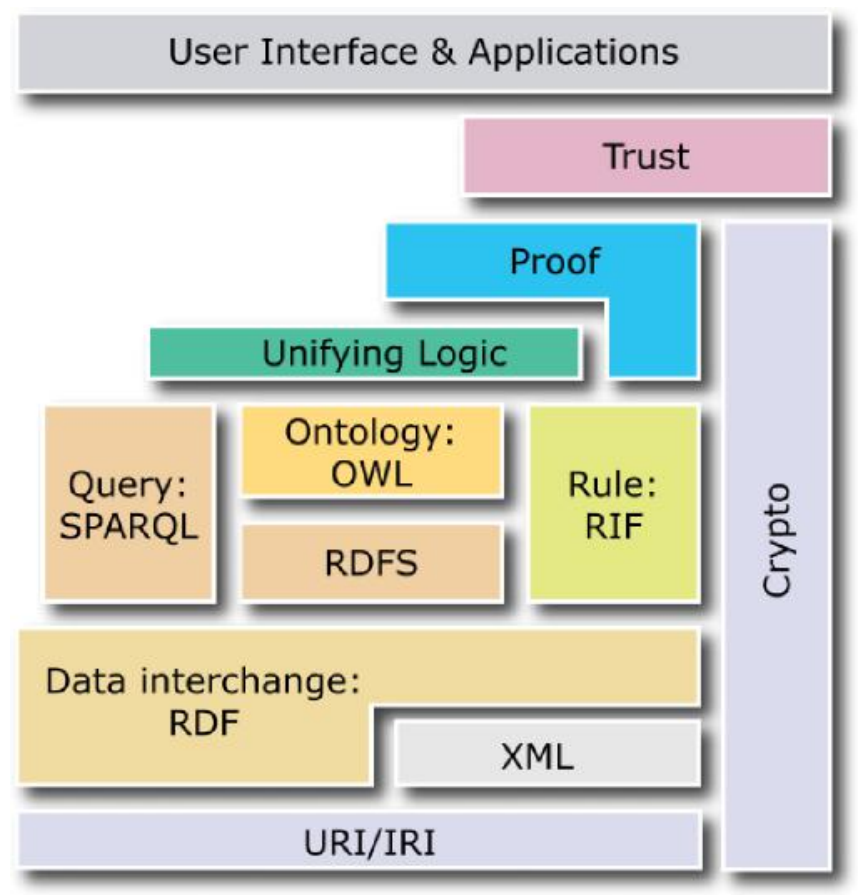

Fig. 1. Semantic web stack [20]

The relevant standards are organized in the Semantic web stack, to which [8] gives an overview. For integrating data and merging knowledge, the use of proper URIs (Uniform resource identifiers) within the ontologies and semantic facts is important. Semantic facts can be exchanged with further systems via the RDF and RDFS standard (Resource Description Framework and schemata). For exchanging, merging and integrating ontologies, OWL is a commonly used standard, which offers extended semantic capabilities. The applied semantic middleware for the presented approach (OntoBroker) is able to support these standards both for import and export. In addition, Ontobroker features its own logic language (ObjectLogic), which is proposed to the $\mathrm{W} 3 \mathrm{C}$ as upcoming standard. SPARQL (SPARQL protocol and RDF 
query language) and RIF (Rule interchange format) were not used in this work, but are accepted by the OntoBroker middleware, as well.

\section{Application for Precision Assembly Lines - Implementation and Verification}

Consistent with Lohse [21] the model of the assembly system is divided into domain concepts suitable for representing the ramp-up of precision assembly systems. The structured model comprises all the information that are not continuously changed during production, representing e.g. the machine model with its sensors, the product definition and corresponding work plan and finally the processes with its capabilities and specifications. However besides modeling the pure static assembly system another layer representing the dynamic changes will be introduced. A mapping functionality correlates the dynamic information with the corresponding structural entities. Besides that also various relationships between structural and/or dynamic entities can be modeled and automatically processed. This is of special importance as in the outlined work this technique was applied for gathering the human experience during ramp-up of precision assembly systems. This all together allows for novel ways of combining information and providing fractions of helpful data to the operators and engineers.

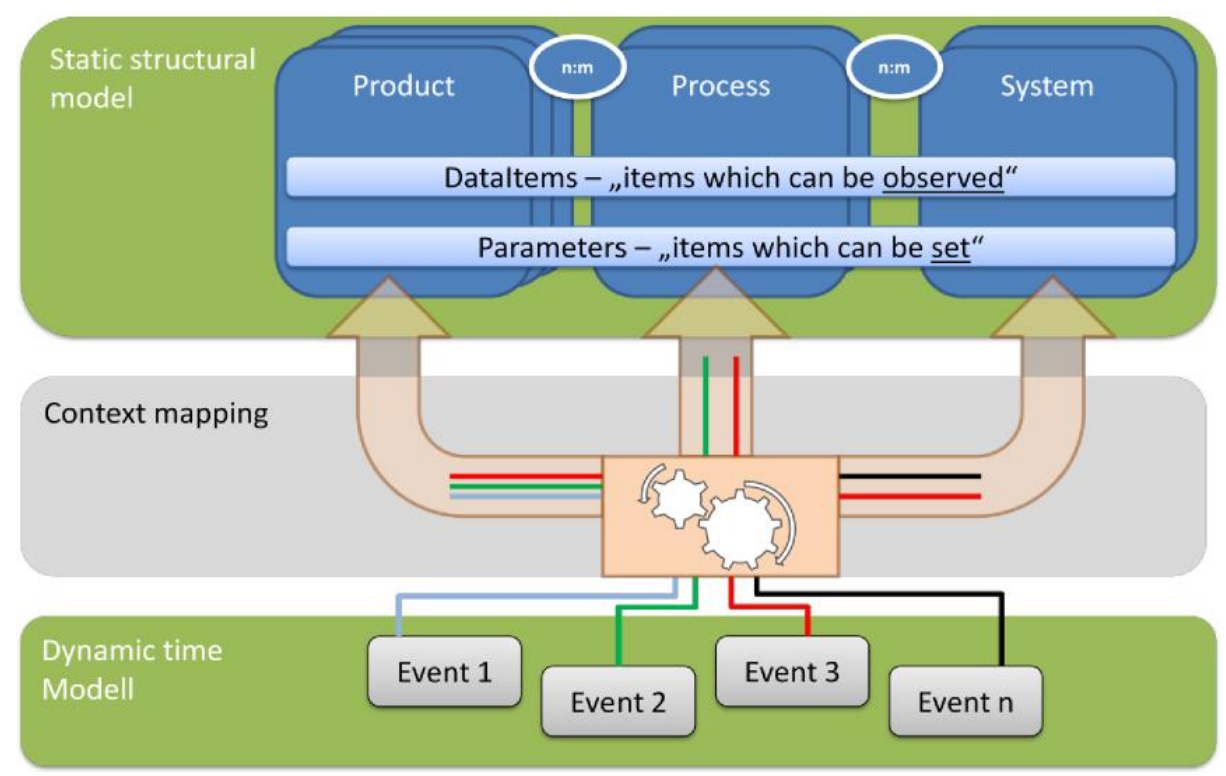

Fig. 2. Three layer design of the implemented system

\subsection{Static Structural Model}

For the static structural model we introduced three domain concepts (Product, Process and System) to represent modular micro assembly lines. 
- Product: A hierarchical representation of the products, its variants and its components (atomic parts of a product) as well as the corresponding product and quality features (e.g. product dimensions or targeted surface quality) are modeled here. The final representation of the product specifications results in a working plan describing the sequence of atomic operations and the parameter set-up to achieve the aimed product variant.

- Process: Here the assembly processes are described in detail. Several different process types are created to categorize the diverse processes according to its capabilities. Within these concepts, the relevant parameters for performing successfull and stable processes are adjusted and dependencies on product features are modeled.

- System: This is a representation of the actual machine, its modules and its sensors. It is a model of the machine "hardware", hierachically ordering the physical structure of the assembly system.

\section{Cross-Domain Definition of Data and Parameters}

Both, 'Parameters' and 'DataItems' are considered to be adjustment or value information most of the times with relation to a physical mechanical setting, a measurement or a corresponding PLC variable. As 'Parameter', all entities are considered, which can be changed by a human operator or a technical installation. As 'DataItem', all entities are considered, which can be read or asked for, but cannot be changed in a direct way. ${ }^{1}$

These concepts are modelled in a cross-domain approach which means that they can be related to each of the already presented domains of the static structural model. For explanation a DataItem representing a sensor holds structural information about in which system or module the sensor is installed but also represents an important monitoring variable for a process.

\subsection{Dynamic Knowledge and Mapping Functionality}

Besides the structural static model also data from dynamic sources is considered. The information is stored in the form of events with a timestamp resulting in a timedriven, event-oriented monitoring system. All events have a unique ID and attributes like event classification main- und subtypes, a primary value and context information.

Depending on their origin and available information these events are mapped to the corresponding structural entities. It is not mandatory to model all possible context information as the system is adaptive enough to handle just as much information as provided. As depicted in Figure 2 the concept allows in contrast to databases also multiple connections. This means that an event can be mapped to multiple atomic attributes of single entities and to multiple domain areas at the same time as well as all possible gradations and mixtures in between.

\subsection{Relationship Model and Reasoning}

As mentioned before the static structural model allows representing different types of relationships between heterogeneous entities on all granularity levels of the domain

\footnotetext{
${ }^{1}$ E.g. a DataItem can also be used as virtual measurement signal combining other information.
} 
model. Also multiple connections with different types between same entities as well as parametrizable relationships are possible. The usage of semantic technologies enables object driven features like inheritance and inference.

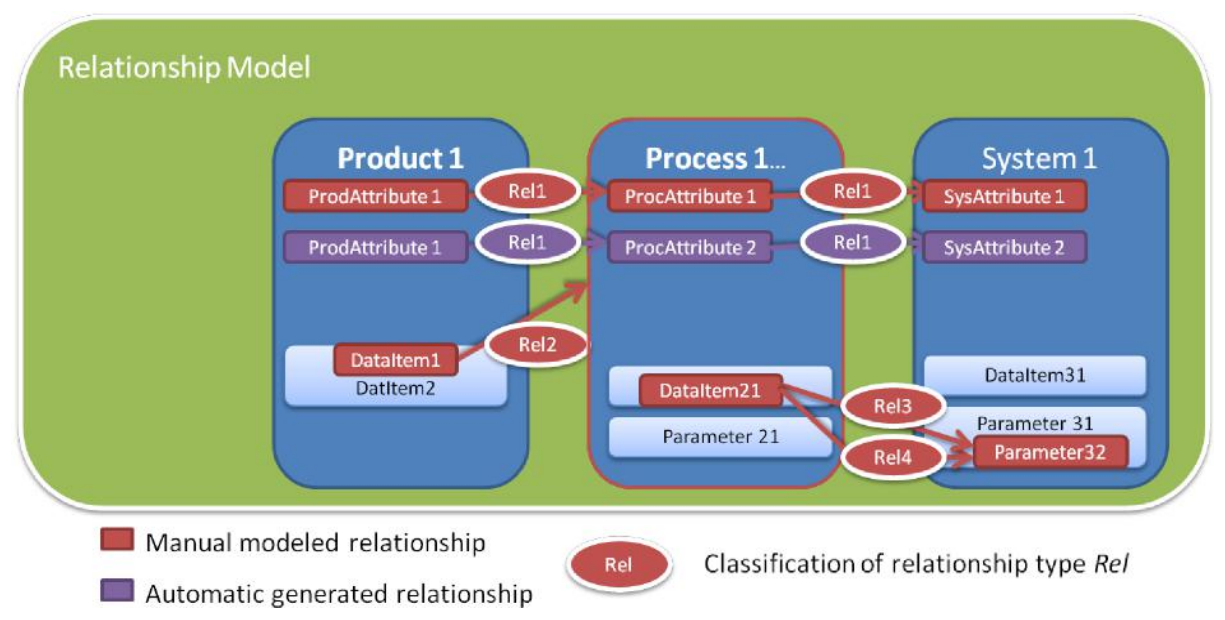

Fig. 3. Example for manual and automated generated relationships

Figure 3 demonstrates some relationship features of the implemented system like the automatic creation of new relationships according to specified patterns (Relation 1), the relation between different granularity levels (Relation 2) and multiple relations even between the same entities (Relation 3 and 4). In summary this realizes a hierarchically structured relationship model which is able to represent the operator experience in a flexible way.

This is of special importance for the gathering the operator observations. It is for example possible to model relations like "failureSource" between an inspection and a processing step. This can prevent the case of extremely time-consuming troubleshooting, which can be in the precision assembly sector a task of several days.

\section{Reasoning}

Besides these object-oriented features pre-defined rules support the automatic extension of the manual defined relations. This can be based on very simple rules or more sophisticated type-specific ones. All rules are modeled object-oriented in the format of extended "if-then" - relationships.

\section{?X [failureSource->?Z] :- ?X [failureSource-> ?Y] and ?Y [failureSource->?Z]}

[Example of of a simple Rule for failureSource propagation written in ObjectLogic]

The presented rule states that if an entity A is a failure source for entity B and B is a failure source for entity $\mathrm{C}$ then $\mathrm{A}$ is also a failure source for $\mathrm{C}$. 


\subsection{Technical Implementation}

The technical implementation of chapters 3.1 to $3 .-3$ was executed during the course of the European project FRAME [22] with industrial partners.

For the ontology modeling the open source software freemind [23] was used for modeling the structure of the machine model (chapter 3.1). With a semantic annotated mindmap [14] which can be automatically translated into semantic languages F-Logic and ObjectLogic (which is proposed to the $\mathrm{W} 3 \mathrm{C}$ as upcoming standard) we realize on the one hand an intuitive system with a technique (mind mapping) which is very familiar for engineers. On the other hand by using the open-source access we also enable additional applications relevant to fulfill production necessities. In the course of the project we implemented e.g. an operator touch-screen for knowledge gathering directly on the shop-floor and a mindmap validation tool to ensure proper input data.

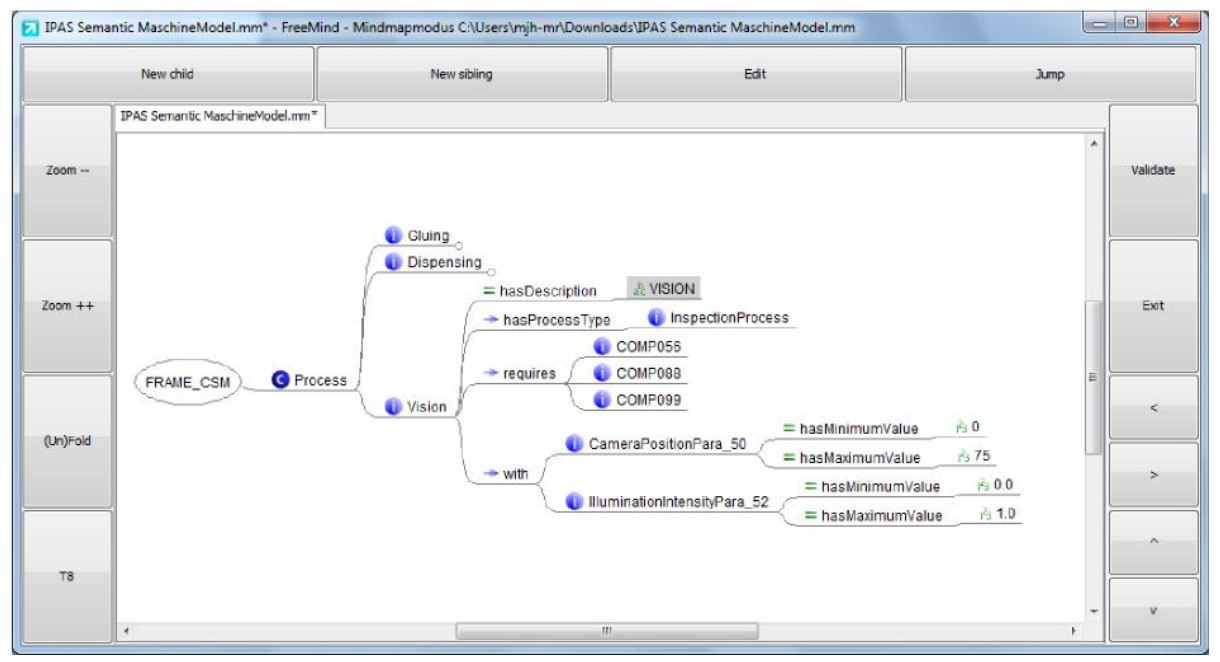

Fig. 4. Screenshot of the implemented touch-screen application; displaying parts of the static structural model with a semantic annotated mindmap

For the presented approach we use the software Ontobroker[24] by the company Ontoprise as semantic middleware which stores all modeled and gathered information inside the internal database. The dynamic knowledge which was described in chapter 3.2 is also fed into it. As Ontobroker is currently one of the best performing semantic systems [25] it is also suitable to store large amount of data which by the nature of micro-assembly production accumulates over the time. The prototypic implementation is able to process over 100 Events per second. In the presented work the semantic middleware was also integrated with Statistical Process Control and HMI input by the operators.

The relationship model (chapter 3.3) was developed in two separate approaches in F-Logic as well as ObjectLogic to enable multiple relations between the entities. Also querying the database is written within these languages. 


\subsection{Verification}

The so implemented system is flexible enough to handle multiple data representation and fulfills the requirements which have been introduced in chapter 1.1.

- Easy gathering of operator knowledge. The workers are able to model their knowledge in a flexible way, providing just the information they think is valuable. Cross-relations between all different entities are possible and not restricted by IT necessities. These connections can also be restricted to certain types or be parameterized. Due to hierarchical structure and rules an automatic creation of connections and therefore an expansion of the assembly model information is realized.

- Flexible context-mapping of static and dynamic data: All gathered dynamic information can be mapped to its structural context in a very human-centered and understandable way. Contrary to databases, features like multiple mappings to different entities with different granularities are possible. There is no need for the involvement of IT experts to model and map new information in the system as this can be done directly by the user.

- Extensibility \& Reusability: The system is implemented completely independent from other software and can due to the time-based information in an event form easily be extended to or integrated with corresponding systems. It is possible to extract the modeled information (or part of it), export it and integrate it in other models.

Special potential lies in the combination between hard, machine-derived data and soft human knowlede and observations. The pre-defined rules realize an costeffective use of of the system. With the presented aproach we are able to combine different data sources in order to provide the shopfloor operators and engineers with helpful data sets to tackle their problems.

\section{Conclusion and Outlook}

During the last years the ramp-up phase has become due to market necessities more and more the focus of research activities. Arising problems are mainly solved through human experience and especially for SMEs there is still a lack of software support. In other industrial applications it has been proven that semantic technologies are suitable for gathering and managing knowledge in a machine-readable format. The presented approach applies semantics to the ramp-up process in the assembly sector and tackles some of the most urgent issues in gathering and reusing operator experience in combination with machine-derived data. The presented work is based on existing standards and offers the possibility for a knowledge management system, which is capable of providing required functionalities to support operators during the ramp-up phase.

Further research in connecting the knowledge management system with existing software such as Manufacturing Execution Systems or Quality Control Software is required. Also the system interface to access the knowledge needs to be adapted to the worker requirements in order to optimal support the ramp-up procedure. The 
presented implementation however provides due to itsflexibility and implemented features already a number of benefits for companies.

The research leading to these results has received funding from the European Community's Seventh Framework Programme (FP7/2007-2013) under grant agreement FRAME n²29208.

\section{References}

1. Sturm, R., Dorner, J., Reddig, K., Seidelmann, J.: Simulation-based evaluation of the ramp-up behavior of waferfabs. In: ASMC 2003, the 14th annual IEEE/SEMI Advanced Semiconductor Manufacturing, pp. 111-117. IEEE, Piscataway (2003)

2. Dombrowski, U., Wesemann, S.: OEE-Kennzahl im Anlagenhochlauf bei KMU: Steigerung der Gesamtanlageneffektivität im Anlagenhochlauf bei KMU mittels der OEEKennzahl, Werkstattstechnik online, vol. 98, pp. 628-633 (2008) (accessed July 28, 2011)

3. Dombrowski, U., Hanke, T.: Lean Ramp-up. Ein Organisationsmodell für den effizienten Serienanlauf in KMU: Lean Ramp-up - An organisation model for efficient series rampups in SME. ZWF Zeitschrift für wirtschaftlichen Fabrikbetrieb 104(10), 877-883 (2009)

4. Fleischer, J., Ender, T., Mössner, A.: Ressourcenmanagement für erfolgreiche Produktionsanläufe. ZWF Zeitschrift für wirtschaftlichen Fabrikbetrieb H. 1-2, S42-S45 (2007)

5. Wiesinger, G., Housein, G.: Schneller Produktionsanlauf von Serienprodukten: Wettbewerbsvorteile durch ein anforderungsgerechtes Anlaufmanagement. Werkstattstechnik Online 92, 505-508 (2002)

6. Berger, U., Thiebus, S.: Wissensmanagement in der Planungsphase: Research concerning knowledge management for development of complex sub-assemblies in the automotive industry. Industrie Management 22(6) Änderungsmanagement, 39-41 (2006)

7. Fleischer, J., Wawerla, M., Nyhuis, P., Winkler, H., Liestmann, V.: Proaktive Anlaufsteuerung von Produktionssystemen entlang der Wertschöpfungskette. Proactive controlling of production ramp-ups along the value chain. Industrie Management $*$ Band 20 (2004) Heft 4, Seite 29-32 20(4), 29-32 (2004)

8. Breslin, J.G., O'Sullivan, D., Passant, A., Vasiliu, L.: Semantic Web computing in industry. Computers in Industry 61(8), 729-741 (2010)

9. W3C, Semantic Web Case Studies and Use Cases (2010), http: / / www.w3 .org/2001/sw/sweo/public/UseCases /

(accessed August 2, 2011)

10. Gruber, T.R.: A translation approach to portable ontology specifications. Knowl. Acquis. 5, 199-220 (1993)

11. Gennari, J.H., Musen, M.A., Fergerson, R.W., Grosso, W.E., Crubézy, M., Eriksson, H., Noy, N.F., Tu, S.W.: The evolution of Protégé: an environment for knowledge-based systems development. Int. J. Hum.-Comput. Stud. 58, 89-123 (2003)

12. Ontoprise, ontoprise: OntoStudio, http://www.ontoprise.de/en/products/ ontostudio/ (accessed August 2, 2011)

13. Ontoprise, ontoprise: Freemind to F-logic (mm2flo), http://www. ontoprise.de/ en/downloads/freemind-to-f-logic-mm2flo/ (accessed August 2, 2011)

14. Busse, J.: Semantic Authoring with semAuth (2011), http: / j jousse.de/semauth/ (accessed August 9, 2011)

15. McBride, B.: Jena: a semantic Web toolkit. IEEE Internet Computing 6(6), 55-59 (2002)

16. Jess, the Rule Engine for the Java Platform, http://www.jessrules.com/ jess/index.shtml (accessed August 2, 2011) 
17. Schmidt, C.: Ontobroker Version 5.1 - Product Documentation (2008), http: / / www. ontoprise.de/fileadmin/user_upload/

Produktdoku_EN/Productdocumentation_OntoBroker_5.1_en.pdf (accessed August 2, 2011)

18. Decker, S., Erdmann, M., Fensel, D., Studer, R.: Ontobroker: Ontology Based Access to Distributed and Semi-Structured Information. In: Meersman, R. (ed.) Database Semantics, Semantic Issues in Multimedia Systems, pp. 351-369. Kluwer Academic Publisher, Boston (1999), ftp: / / ftp.aifb.uni-karlsruhe.de/pub/mike/dfe/paper/rdf3.ps

19. Berners-Lee, T., Hendler, J., Lassila, O.: The Semantic Web. Scientific American 284(5), 34-43 (2001)

20. W3C, Semantic Web Stack (2007),

http: / /www.w3 .org/2007/03/layerCake.png (accessed August 2, 2011)

21. Lohse, N., Hirani, H., Ratchev, S.: Equipment ontology for modular reconfigurable assembly systems: Methodisches Konzept für die Umgestaltung modularer Fertigungseinrichtungen von Montagesystemen. International Journal of Flexible Manufacturing Systems 17(4), 301-314 (2005)

22. FRAME. EU FP7 Project (2011), http://www. frame-eu.org/ (accessed October 18, 2011)

23. FreeMind (2011), http://freemind.sourceforge.net/wiki/index.php/ Main_Page (accessed October 17, 2011)

24. Schmidt, C.: Ontobroker Version 5.1 - Product Documentation (2008), http: / / www.ontoprise.de/fileadmin/user_upload/ Produktdoku_EN/Productdocumentation_OntoBroker_5.1_en.pdf (accessed August 2, 2011)

25. OpenRuleBench - Benchmarks for Semantic Web Rule Engines (2011), http: / / rulebench.projects. semwebcentral .org/ (accessed October 19, 2011) 